\title{
Simultaneous irradiation of tungsten with deuterium and carbon at elevated
}

\section{temperatures}

\author{
H.T. Lee* and K. Krieger \\ Max-Planck-Institut für Plasmaphysik, EURATOM Association, Boltzmannstr. 2, 85748 Garching, \\ Germany
}

\begin{abstract}
The dynamics of $\mathrm{W}$ sputtering as well as the accumulation of implanted $\mathrm{C}$ for $\mathrm{W}$ layers deposited on Ni substrates under simultaneous $12 \mathrm{keV} C_{2}^{-}$and $9 \mathrm{keV} D_{3}^{+}$ion irradiation was studied in-situ by ion beam analysis using $2.5 \mathrm{MeV}^{3} \mathrm{He}^{+}$ions. Specifically, the change in the W and $\mathrm{C}$ areal densities as a function of the $\mathrm{C}$ fraction in the incident total flux $\left(f_{\mathrm{C}}\right)$ and temperature $\left(400-600^{\circ} \mathrm{C}\right)$ is discussed. C-only irradiation leads to increased C self-sputtering with increasing temperature. Simultaneous irradiations at RT indicate contributions of chemical erosion in the W sputtering regime, which are not observed at elevated temperatures. The increased C selfsputtering dominates the implantation sputtering behavior at elevated temperatures, shifting the transition point between continous $\mathrm{W}$ sputtering and continous $\mathrm{C}$ layer growth to higher $f_{\mathrm{C}}$. The effect of D is observable only with the onset of C layer buildup, but chemical erosion by D could not be distinguished from kinematic effects in the $\mathrm{C}$ deposition regime.
\end{abstract}

PACS: 28.52.Fa, 79.20.Rf, 61.80.Jh, 61.82.Bg

JNM Keywords: Tungsten, Carbon, Hydrogen, Ion irradiation, Radiation effects

PSI-18 Keywords: Tungsten, Sputtering, Carbon impurities, Ion-surface interactions, Surface analysis

*Corresponding author address: Boltzmannstraße2, D-85748 Garching, Germany.

*Corresponding and presenting author email: heun.lee@ipp.mpg.de

Presenting author: H.T. Lee 


\section{Introduction}

The use of carbon (C) strike plates located directly adjacent to tungsten (W) tiles in ITER leads to C impurities in the incident flux of fuel ions [1]. The ASDEX-Upgrade tungsten divertor experiments have shown that $\mathrm{W}$ erosion is dominated by multiply charged $\mathrm{C}$ impurities accelerated in the sheath potential [2]. The resulting simultaneous bombardment of W surfaces with both gaseous fuel and $\mathrm{C}$ impurity ions result in a competition of erosion and deposition processes that is significantly different from pure hydrogen or C-only bombardment [3]. Of particular importance and application to fusion research is the prediction of the transition point from erosion to deposition as it separates areas of continuous erosion from areas of C layer formation. Significant deviation in the prediction of this transition point from comparing RT experimental results with simulations exist [4]. In particular, there is still an uncertainty in distinguishing the contribution of surface roughness from chemical erosion in RT measurements [5]. With increasing temperature, increased $\mathrm{W}$ sputtering is observed due to decrease in C layer formation rate [3]. However, to this date, no experiments have conclusively shown a clear correlation between $\mathrm{C}$ chemical erosion in the $\mathrm{W}$ matrix and its effect on $\mathrm{W}$ sputter yield and $\mathrm{C}$ concentration. Here we present results for dynamic $\mathrm{W}$ erosion and $\mathrm{C}$ layer growth due to simultaneous irradiation by combined $\mathrm{C}_{2}{ }^{-}-\mathrm{D}_{3}{ }^{+}$ions at RT and $400-600^{\circ} \mathrm{C}$.

\section{Experiments}

W layers of thickness $380 \mathrm{~nm}$ - $410 \mathrm{~nm}$ were deposited on polished Ni substrates using a magnetron sputter device. Fig. 1a shows the image of a virgin W layer obtained by atomic force microscopy (AFM). The Rq, or root mean squared roughness, representing the standard deviation of the distribution of surface heights was $3.6 \mathrm{~nm} \pm 0.4 \mathrm{~nm}$. Annealing to $600 \mathrm{C}$ increases the $\mathrm{Rq}$ to $8.2 \mathrm{~nm} \pm 0.8 \mathrm{~nm}$; a factor of $\sim 2$ increase in comparison to the initial surface. However, both 
roughness values are smaller than the calculated mean projectile range of $C$ and $D$ at $10-15 \mathrm{~nm}$, and 40-50 nm, respectively. Therefore, the initial surface of specimens at all temperatures can be assumed to be smooth.

A dual-beam ion accelerator system [6] was used to implant $12 \mathrm{keV} \mathrm{C}_{2}{ }^{-}$and $9 \mathrm{keV} \mathrm{D}_{3}{ }^{+}$ with fluxes of $7-10 \times 10^{17} \mathrm{C}^{-} / \mathrm{m}^{2} \mathrm{~s}$ and $10^{18}-10^{19} \mathrm{D}^{+} / \mathrm{m}^{2} \mathrm{~s}$ up to fluences of $\sim 10^{22} \mathrm{C}^{-} / \mathrm{m}^{2}$ and $\sim 10^{23}$ $\mathrm{D}^{+} / \mathrm{m}^{2}$. It was assumed that each species has an incidence energy of $6 \mathrm{keV}$ and $3 \mathrm{keV}$, respectively. The angle of incidence for both ion species was $15^{\circ}$ to the surface normal. To obtain the different $\mathrm{C} / \mathrm{D}$ ratios, the $\mathrm{C}$ flux was kept nearly constant (factor of 2) while the $\mathrm{D}$ flux was varied. The specimens were heated using a ceramic heater located behind the specimens to 400 , 500 , and $600^{\circ} \mathrm{C}$. The temperature was measured using a chromel-alumel thermocouple as well as a two wavelength pyrometer. Ion beam analysis with $2.5 \mathrm{MeV}^{3} \mathrm{He}^{+}$ions was performed in-situ between irradiation sessions, providing information of the dynamic evolution of surface composition with increasing fluence. Rutherford back-scattering spectrometry (RBS) was used to measure the change of $\mathrm{W}$ areal density by calculating the difference in the $\mathrm{W}$ layer thickness. The main error source is the mixing of the $\mathrm{W}-\mathrm{Ni}$ interface with increasing temperature, resulting in an uncertainty of $<10 \%$. The amount of implanted $C$ was measured using the nuclear reaction ${ }^{12} \mathrm{C}\left({ }^{3} \mathrm{He}, \mathrm{p}\right){ }^{14} \mathrm{~N}$ [8]. These measurements were calibrated using a reference a-C:D layer with known $\mathrm{C}$ areal densities with a resulting accuracy $<10 \%$. Further details of the measurement technique can be found in Ref [6].

A new diagnostic tool, the Beam viewing system (BVS) [9] capable of imaging the ion beams using a microchannel plate and phosphor screen was used to obtain quantitative information of the incident beam distributions, the C/D ratio, as well as locating the analysis spot of the ${ }^{3} \mathrm{He}^{+}$ions. Current measurements using a picoammeter were used to calibrate the beam intensities. 


\section{Results and Discussion}

\subsection{C-only irradiations at $R T, 400,500$, and $600{ }^{\circ} \mathrm{C}$}

The results of C-only irradiation for four different temperatures are plotted in Fig. 2 as implanted C against sputtered $\mathrm{W}$. This parametric representation of the data allows clear distinction between continous $\mathrm{W}$ erosion and $\mathrm{C}$ deposition regimes [5], while the effects of temperature on the behavior of sputtering and implantation can also be seen. Fig. 2 shows that for all temperatures a buildup of $\mathrm{C}$ layer occurs, indicative of the $\mathrm{C}$ deposition regime which protects the underlying $\mathrm{W}$ from further sputtering. However, a temperature dependence of increased W sputtering with increasing temperature is observed. This trend results from increased loss of C from the surface and can be explained by $\mathrm{C}$ diffusion, an increase in surface roughness, or C selfsputtering. Since C diffusion below $700 \mathrm{C}$ is very small [3], its effect is considered negligible under present experimental conditions.

Initially, no temperature dependence is observed in the implantation sputtering curves, as the data is well fitted by the simulated TRIDYN [10] curve. However, when $\sim 0.1 \times 10^{22} \mathrm{~m}^{-2}$ of $\mathrm{W}$ is sputtered ( $15 \mathrm{~nm}$ assuming pure W), a temperature dependence is seen. Clearly this is when the implanted $\mathrm{C}$ is exposed, leading to the interaction of the incident $\mathrm{C}$ ions with a mixed $\mathrm{W}-\mathrm{C}$ surface. Here, we define the surface as meaning the region of interaction between the implanted ions. The observed temperature effect is then an indication that the mixed W-C surface proceeds to develop rather differently at different temperatures. Specifically, there is an increased loss of C from the surface, which can be explained by the increase in C self-sputtering with temperature [3]. Using the exponential fit for the temperature dependent C self-sputtering yield in Ref. [3], one obtains lower C-C surface binding energies (SBEs) of 5.16eV, 4.50eV, and $3.89 \mathrm{eV}$ for 400, 500, and $600^{\circ} \mathrm{C}$, respectively. Implementing the SBE for $600^{\circ} \mathrm{C}$ in TRIDYN however, fails to fit the 
experimental data; see Fig. 2. Clearly, the effect of increased C self-sputtering by itself is inadequate to explain the observed trends. An AFM image of the $\mathrm{C}$ irradiation spot at $600^{\circ} \mathrm{C}$ is shown in Fig. 1b. The surface roughness has increased to $\mathrm{Rq}=48.4 \mathrm{~nm} \pm 5.5 \mathrm{~nm}$, which is comparable to the mean ion ranges of the incident ions. An increase in surface roughness results in increased $\mathrm{W}$ sputtering by broadening of the distribution of local incidence angles. This is illustrated by the TRIDYN simulation curve using a larger incidence angle of 35 degrees to approximate "roughness" [5], which fits the $600^{\circ} \mathrm{C}$ trend better. It is postulated that the increase in $\mathrm{C}$ self-sputtering hinders the growth of the protective $\mathrm{C}$ layer by preferential sputtering of $\mathrm{C}$, resulting in a rougher surface that causes increased $\mathrm{W}$ sputtering. Therefore, we have observed for the first time, temperature dependent $\mathrm{C}$ self-sputtering from a mixed $\mathrm{W}-\mathrm{C}$ surface, that controls the rate of $\mathrm{C}$ buildup and consequently the amount of $\mathrm{W}$ sputtered at elevated temperatures.

\subsection{Simultaneous $\mathrm{C}_{2}^{-}-\mathrm{D}_{3}{ }^{+}$irradiations at $R T$}

In general, simultaneous $\mathrm{C}_{2}^{-}-\mathrm{D}_{3}{ }^{+}$irradiations of $\mathrm{W}$ results in one of two principal regimes where either $\mathrm{W}$ erosion or $\mathrm{C}$ deposition dominates depending on the $\mathrm{C}$ fraction in the total incident flux $\left(f_{\mathrm{C}}\right)[4]$. In the $\mathrm{W}$ erosion regime, the surface remains a mixture of $\mathrm{W}$-C, while in the $\mathrm{C}$ deposition regime, a pure $\mathrm{C}$ layer forms over the mixed $\mathrm{W}$-C surface. The implantation and sputtering curves for simultaneous $\mathrm{C}_{2}^{-}-\mathrm{D}_{3}{ }^{+}$irradiations at RT with TRIDYN simulations are plotted in Fig. 3a. Experiments at $f_{C}=11-25 \%$ results in the $C$ deposition regime, and are well fitted with TRIDYN simulations, indicating that kinematic effects dominate. For $f_{C}<11 \%$, the experimental results show a steady state $\mathrm{W}$ sputtering regime, where the amount of $\mathrm{C}$ stays constant while $\mathrm{W}$ is continuously sputtered. Therefore, the transition point at RT is expected to be between $7 \%<f_{C}<11 \%$ which is lower than the previously reported $f_{C} \approx 15 \%$ [4]. For $f_{C}=7 \%$ 
however, no increase in surface roughness is observed, but TRIDYN simulation still deviates from the experimental data. Specifically, the amount of implanted $C$ is $\sim 70 \%$ less than the predicted amount. In a steady state $\mathrm{W}$ sputtering regime, the $\mathrm{C}$ amount depends only on the $\mathrm{C} / \mathrm{D}$ ratio in the incident flux. The good agreement between the higher $f_{C}$ cases with simulations indicates that the BVS is able to measure the $\mathrm{C} / \mathrm{D}$ ratio accurately. Therefore, for $f_{C}=7 \%$, the difference in TRIDYN simulation from experiment is attributed to the non-kinematic effect of chemical erosion.

\subsection{Simultaneous $\mathrm{C}_{2}^{-}-\mathrm{D}_{3}{ }^{+}$irradiations at 400,500 , and $600{ }^{\circ} \mathrm{C}$}

The implantation and sputtering curves for simultaneous $\mathrm{C}_{2}^{-}-\mathrm{D}_{3}{ }^{+}$irradiations at 400,500 , and $600{ }^{\circ} \mathrm{C}$ are plotted in Figs. 3b-d. TRIDYN simulations for $f_{C}=25 \%$ and $f_{C}=7 \%$ from Fig. 3a, along with C-only irradiation curves from Fig. 2 are also plotted as reference to measure the effect of D. A shift of the transition point is observed for 500 and $600^{\circ} \mathrm{C}$, with the transition point now between $11 \%<f_{C}<14 \%$ and $f_{C}>14 \%$, respectively. At $f_{C}$ below the transition point, the implantation sputtering behaviors can be fitted with TRIDYN simulation; see $f_{C}=7 \%$ in Figs $3 b-d$. However, the experimental C amounts are $~ 30 \%$ lower than the predicted steady state TRIDYN value. This discrepancy may be interpreted as chemical erosion like discussed in Section 3.2, but then it should exhibit temperature dependence. Chemical erosion of a mixed W-C surface decreases with increasing temperature [11]. However as seen from Fig. 4a, no temperature dependence is seen in the amount of implanted $C$ for $f_{C}=7 \%$ between 400,500 , and $600^{\circ} \mathrm{C}$. For $f_{C}=11 \%$, there is a clear reduction in $\mathrm{C}$ amount with increasing temperature, which is opposite to the trend for chemical erosion. This $\mathrm{C}$ reduction is due to the increase in $\mathrm{C}$ self-sputtering yield as discussed in Sec. 3.1, and chemical effects appear very limited. Also, in Fig. 3d, the implantation sputtering behavior is nearly independent of the increase in $D$ between $7 \%<f_{C}<14 \%$, further 
supporting the limited contribution of chemical erosion effects. The difference between experiments and simulation may arise due to surface roughness effects but nevertheless, in a W sputtering regime at elevated temperatures, $\mathrm{C}$ self-sputtering appears to be the dominant mechanism for $\mathrm{C}$ removal with negligible contribution from $\mathrm{D}$.

At $f_{C}$ above the transition point, the effect of $D$ is strongly observed after $0.15 \sim 0.2 \times 10^{22} \mathrm{~m}^{-2}$ of implanted C. For example, compare $f_{C}=100 \%$ with $f_{C}=14 \%$ in Fig. 3b or $f_{C}=100 \%$ with $f_{C}$ $=25 \%$ in Figs. 3c and 3d. This appears to be the critical C amount necessary from which point the buildup of $\mathrm{C}$ layer develops. Increasing the $\mathrm{D}$ flux reduces the rate of the $\mathrm{C}$ layer buildup but it is not possible to separate chemical effects from the kinematic component by comparing to TRIDYN results. The reason, as discussed in Section 3.1, is that TRIDYN cannot properly account for the increase in $\mathrm{C}$ self-sputtering at elevated temperatures. However, in Fig. 4d, for both $f_{C}=14 \%$ and $f_{C}=25 \%, C$ amount decreases with increasing temperature. Since the chemical erosion of a W-C surface decrease with temperature [11], the large decrease in implanted C with increasing temperature demonstrates the limited chemical effect of D. Therefore, C selfsputtering appears to be the dominant mechanism in $\mathrm{C}$ removal and $\mathrm{W}$ sputtering in simultaneous irradiations at elevated temperatures.

\section{Conclusions}

The importance of temperature on the implantation sputtering behavior of C-only and simultaneous $\mathrm{C}_{2}{ }^{-}-\mathrm{D}_{3}{ }^{+}$irradiations have been shown. First, for C-only irradiations, an increase in C self-sputtering in a mixed W-C surface has been observed with increasing temperature. TRIDYN simulation with SBE derived from $C$ self-sputtering yields in the literature was found to underestimate the amount of $\mathrm{W}$ sputtered when compared to experimental data. However, surface 
roughness also increased, possibly from preferential sputtering of $C$ that enhanced the amount of W sputtered.

Secondly, for simultaneous irradiations at elevated temperatures, the transition point from W sputtering to $\mathrm{C}$ deposition was seen to shift to higher $f_{\mathrm{C}}$ with increasing temperature. In the $\mathrm{W}$ sputtering regime, chemical erosion by D ions is observed only at RT with no temperature dependence seen at the higher temperatures. This indicates that chemical erosion is negligible for a mixed $\mathrm{W}-\mathrm{C}$ surface in the steady state $\mathrm{W}$ sputtering regime. In the $\mathrm{C}$ deposition regime, the influence of D is small compared to the increased C self-sputtering with temperature. Therefore, the $\mathrm{C}$ self-sputtering has a larger impact in the implantation sputtering behavior of a mixed W-C system under simultaneous irradiation than D chemical effects at elevated temperatures.

\section{Acknowledgements}

The authors would like to thank C. Adelhelm for AFM measurements; P. Matern, G. Matern, M. Fusseder, J. Dorner for valuable technical assistance. 


\section{References}

[1] K. Schmid, K.Krieger, et al., J. Nucl. Mater. 363-365 (2007) 674.

[2] K. Krieger et al., J. Nucl. Mater. 266-269 (1999) 207.

[3] K. Schmid, J. Roth, J. Nucl. Mater. 313-316 (2003) 302.

[4] I. Bizyukov, et al., J. Nucl. Mater. 363-365 (2007) 1184-1189.

[5] I. Bizyukov, K. Krieger, et al., J. Appl. Phys. 100 (2006) 113302.

[6] I. Bizyukov, K. Krieger. Rev. Sci. Instrum. 77 (2006) 043501.

[7] S.Y. Tong, W.N. Lennard, et al., Nucl. Instrum. Methods B45 (1990) 41.

[8] I. Bizyukov, K. Krieger, et al., J. Nucl. Mater. 337-339 (2005) 965.

[9] J.L. Forand, C. Timmer, E. Wahlin, et. al., Rev. Sci. Instrum. 61 (1990) 3372.

[10] W. Moeller, W. Eckstein, J.P. Biersack, Comput. Phys. Commun. 51 (1998) 355.

[11] M. Balden, E. de Juan Padro, et al., J. Nucl. Mater. 337-339 (2005) 980. 


\section{Figure Captions}

Figure 1: $\quad$ Atomic force microscope images of: (a) Virgin specimen before heat treatment; (b) C irradiated spot at $600{ }^{\circ} \mathrm{C}$.

Figure 2: $\quad$ Implantation sputtering curves for C-only irradiation as a function of temperature with TRIDYN simulations for surface roughness approximation [5] and increased C self-sputtering using reduced SBE derived from Ref. [3].

Figure 3: $\quad$ Implantation sputtering curves for simultaneous $\mathrm{C}_{2}{ }^{-}-\mathrm{D}_{3}{ }^{+}$irradiations at: (a) RT; (b) $400{ }^{\circ} \mathrm{C}$; (c) $500{ }^{\circ} \mathrm{C}$; (d) $600{ }^{\circ} \mathrm{C}$, plotted for varying $f_{C}$.

Figure 4: Implantation sputtering curves for simultaneous $\mathrm{C}_{2}{ }^{-}-\mathrm{D}_{3}{ }^{+}$irradiations in: (a) W sputtering regime and; (b) $\mathrm{C}$ deposition regime, as a function of temperature and $f_{C}$. 

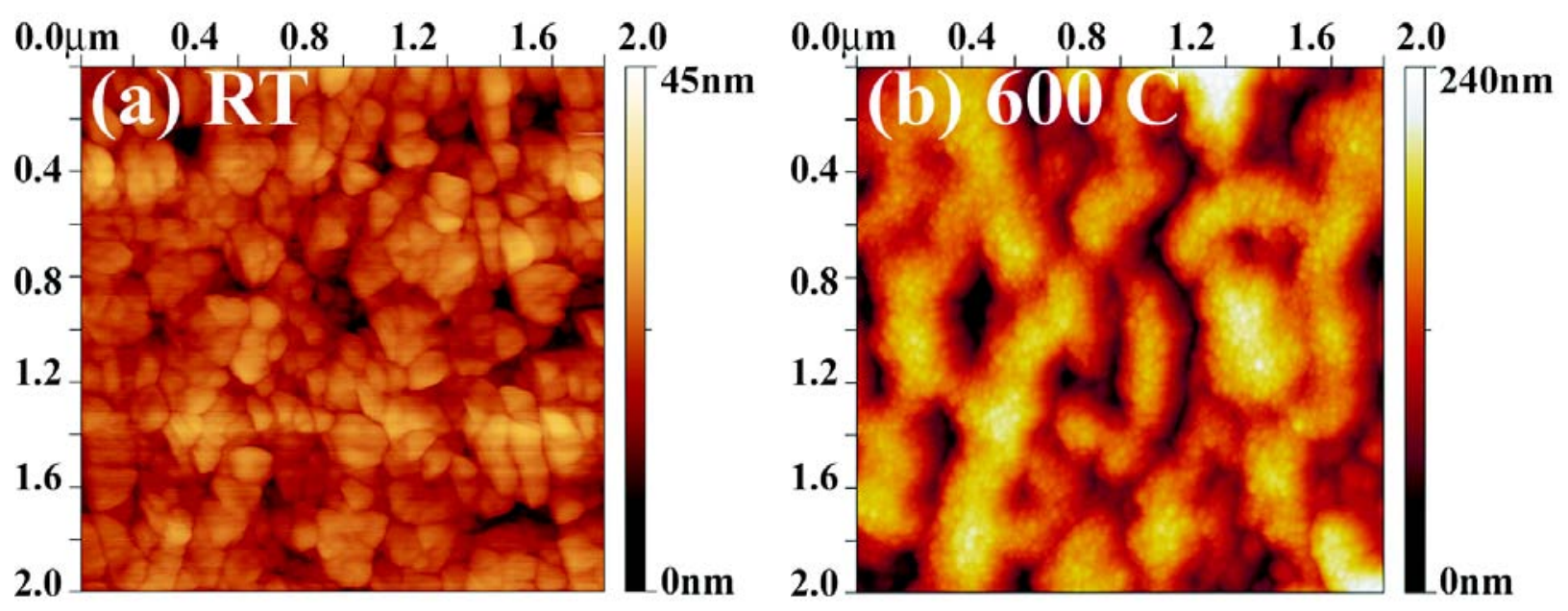

Figure 1 


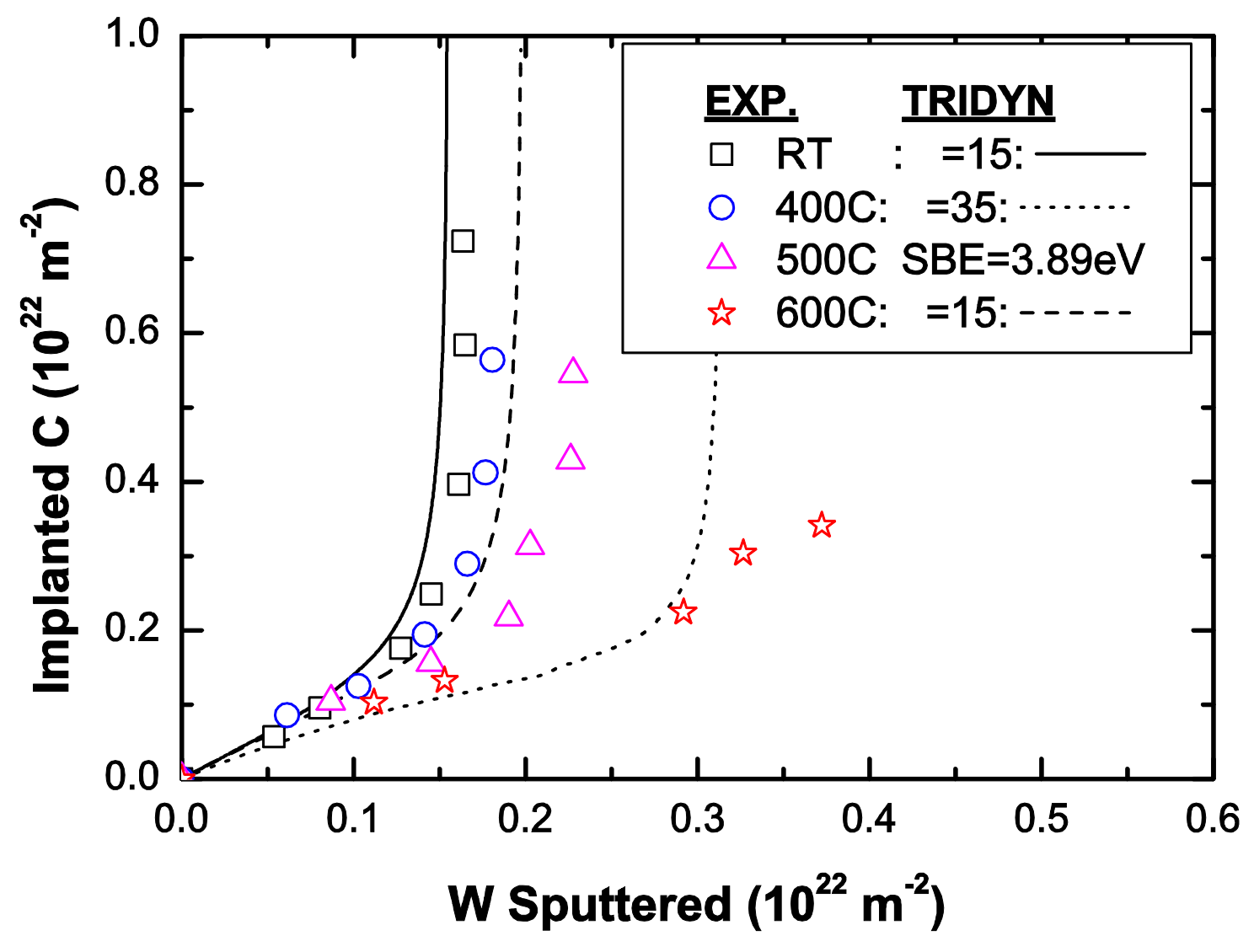

Figure 2 


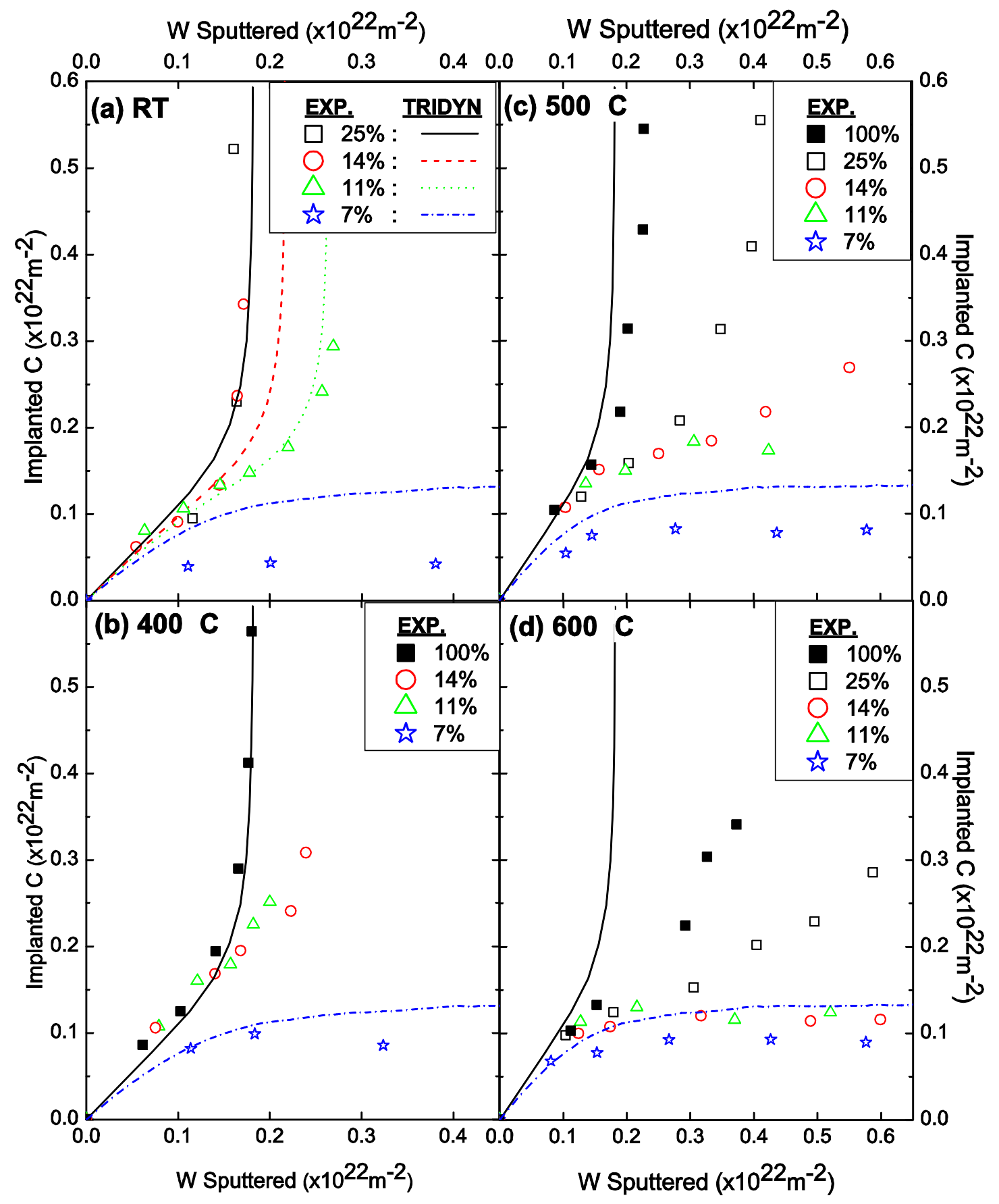

Figure 3 


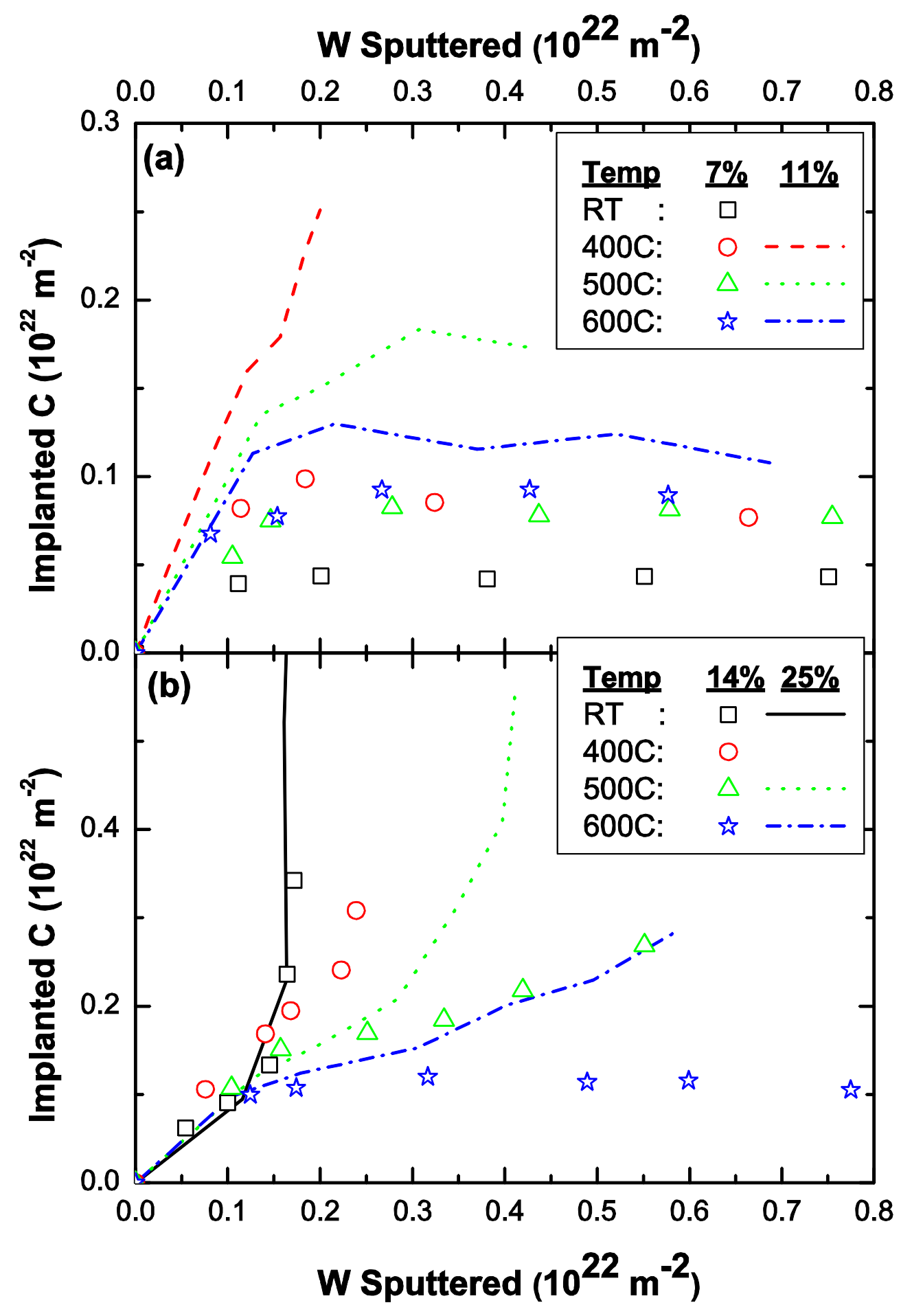

Figure 4 